causative role to arginase will depend on the results of the genetic studies that Zimmermann and colleagues' work warrants. Do single nucleotide polymorphisms in human arginase dysregulate expression and/or function so as to contribute to asthma pathogenesis? The role of arginase in the realm of asthma will ultimately be dictated by the answer to this question.

1. 2003. Asthma Prevalence, Health Care Use and Mortality, 2000-2001. National Center for Health Statistics, Center for Disease Control.

2.Zimmermann, N., et al. 2003. Dissection of experimental asthma with DNA microarray analysis identifies arginase in asthma pathogen- esis. J. Clin. Invest. 111:1863-1874 doi:10.1172/JCI200317912.

3. Morris, S.M., Jr. 2002. Regulation of enzymes of the urea cycle and arginine metabolism. Annu. Rev. Nutr. 22:87-105.

4. Fischer, A., Folkerts, G., Geppetti, P., and Groneberg, D.A. 2002. Mediators of asthma: nitric oxide. Pulm. Pharmacol. Ther. 15:73-81.

5. Wechsler, M.E., et al. 2000. Exhaled nitric oxide in patients with asthma: association with NOS1 genotype. Am. J. Respir. Crit. Care Med. 162:2043-2047.

6. Endo, M., et al. 2003. Induction of arginase I and II in bleomycin induced fibrosis of mouse lung. Am. J. Physiol. Lung Cell. Mol. Physiol. doi:10.1152/ ajplung.00434.2002.

7. Meurs, H., et al. 2002. Increased arginase activity underlies allergen-induced deficiency of cNOSderived nitric oxide and airway hyperresponsiveness. Br. J. Pharmacol. 136:391-398.

8. Wei, L.H., Jacobs, A.T., Morris, S.M.J., and Ignarro, L.J. 2000. IL-4 and IL-13 upregulate arginase I expression by cAMP and JAK/STAT6 pathways in vascular smooth muscle cells. Am. J. Physiol. Cell. Physiol. 279:C248-C256.

9. Chang, C., Zoghi, B., Liao, J.C., and Kuo, L. 2000. The involvement of tyrosine kinases, cyclic $\mathrm{AMP} /$ protein kinase A, and $\mathrm{p} 38$ mitogen-activated protein kinase in IL-13-mediated arginase I induction in macrophages: its implications in IL-13-inhibited nitric oxide production. J. Immunol. 165:2134-2141.

10. Mowen, K.A., et al. 2001. Arginine methylation of STAT1 modulates IFNalpha/beta-induced transcription. Cell. 104:731-741.

11. Liu, B., Gross, M., ten Hoeve, J., and Shuai, K 2001. A transcriptional corepressor of Stat 1 with an essential LXXLL signature motif. Proc. Natl. Acad. Sci. U. S. A. 98:3203-3207.

12. Shi, O., Morris, S.M.J., Zoghbi, H., Porter, C.W., and O'Brien, W.E. 2001. Generation of a mouse model for arginase II deficiency by targeted disruption of the arginase II gene. Mol. Cell. Biol. 21:811-813.

13. Iyer, R.K., et al. 2002. Mouse model for human arginase deficiency. Mol. Cell Biol. 22:4491-4498.

\title{
An eye on insulin
}

\author{
Sarah K. Bronson, ${ }^{1}$ Chad E.N. Reiter, ${ }^{1}$ \\ and Thomas W. Gardner ${ }^{1,2}$
}

${ }^{1}$ Department of Cellular and Molecular Physiology, and

${ }^{2}$ Department of Ophthalmology, Penn State College of Medicine, Hershey,

Pennsylvania, USA

Diabetic retinopathy, the most frequent complication of diabetes and leading cause of vision loss, involves vascular and neural damage in the retina. Insulin and IGF-1 signaling are now shown (see the related article beginning on page 1835) to contribute to retinal neovascularization, in part, by modulating the expression of various vascular mediators.

J. Clin. Invest. 111:1817-1819 (2003). doi:10.1172/JCI200318927.

Physiologic and pathologic blood vessel growth patterns are stimulated by local and systemic influences. The hope of angiogenesis research is to understand these complex interactions in order to provide better means to control pathologic vessel forma-

\footnotetext{
Address correspondence to: Thomas W. Gardner, Department of Cellular and Molecular Physiology, and Department of Ophthalmology, Penn State College of Medicine, 500 University Drive, Hershey, Pennsylvania 17033, USA.

Phone: (717) 531-6711;

Fax: (717) 531-7667;

E-mail: tgardner@psu.edu.

Conflict of interest: The authors have declared that no conflict of interest exists. Nonstandard abbreviations used: retinopathy of prematurity (ROP); diabetic retinopathy (DR); hypoxia-inducible factor-1 (HIF-1); endothelin-1 (ET-1); insulin receptor (IR); insulin-like growth factor-1 receptor (IGF-1R), vascular endothelial insulin receptor knockout (VENIRKO); vascular endothelial insulin-like growth factor receptor knockout (VENIFARKO).
}

tion, or perhaps stimulate appropriate vessel growth, to reduce maladaptive consequences. In the retina, normal vessel growth occurs in the plane of the retina from the optic nerve toward the periphery in a radial pattern and is guided by cues from astrocytes in the inner retina (1). This growth is mediated by VEGF and other ligands (2), while angioblasts from the circulation can provide endothelial progenitors (3).

\section{The problem of retinal neovascularization}

Pathologic neovascularization of the retina is a common and serious complication of retinopathy of prematurity (ROP) and diabetic retinopathy (DR). The treatment of DR, ablation of the diseased retina with laser photocoagulation or cryotherapy to cause involution of the new vessels, has remained fundamentally unchanged for almost 50 years. The nature of the growth promoting stimuli is not well understood, but in ROP the stimulus is assumed to be in part due to perinatal retinal hyperoxia followed by hypoxia. The classic response to hypoxia includes hypoxiainducing factor-1 (HIF-1) translocation to the nucleus and subsequent downstream events such as the upregulation of VEGF, eNOS, and endothelin-1 (ET-1). The fact that VEGF is increased in the vitreous of diabetic patients makes it tempting to speculate that diabetes induces a hypoxic, or HIF-1-driven response that is similar to that observed in ROP. Current animal models of diabetes do not develop proliferative retinopathy and the only model that simulates the neovascularization seen clinically is that induced by relative hypoxia in developing retinas. Postnatal mice are placed in hyperoxic conditions for several days, at a time when their vessels have not yet reached the peripheral retina, which causes vasoconstriction; when they are returned to room air, the vasoconstriction is relieved and neovascularization develops when the retina perceives relative hypoxia (4). This model provides a rodent model of neovascularization in the absence of systemic metabolic defects due to insulin depletion or resistance. Smith et al. (5) previously showed that an IGF-1 inhibitor blocked neovascularization in this model but no studies have examined the effect of the insulin receptor. 


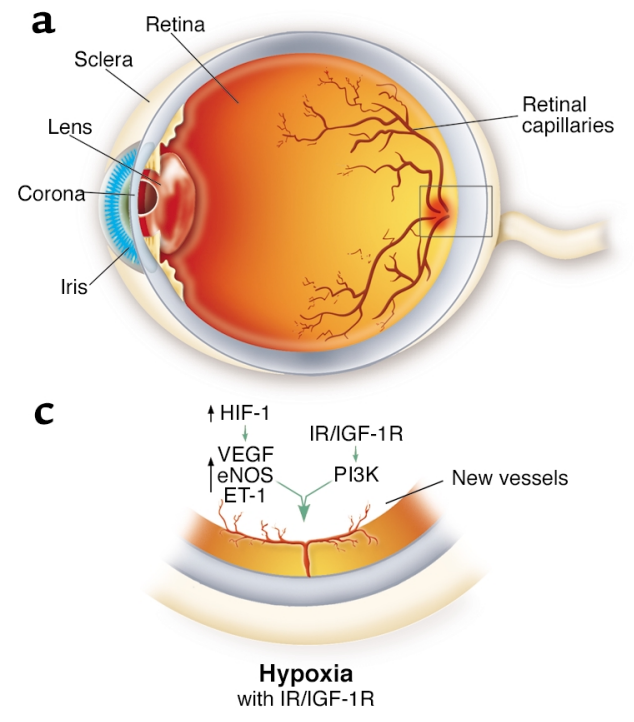

b

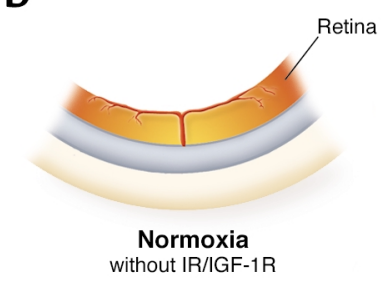

d

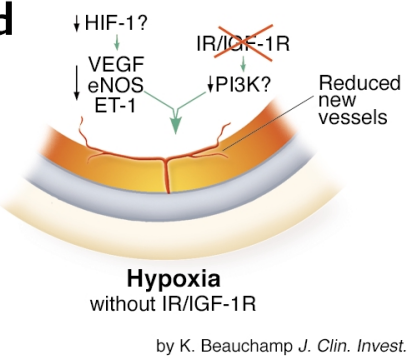

\section{Figure 1}

Retinal neovascularization is the result of hypoxia-induced damage to the neural retina and its capillaries (a). Following investigation of the effects of hypoxia in the presence and absence of IR/IGF-1R on retinal vascularization, Kondo et al. (6) found that under normoxic condition (b), the retinas of mice develop normally in the absence of endothelial IR/IGF-1R. Presumably, sufficient growth factors (e.g., VEGF) are present to facilitate normal development. Under conditions of relative hypoxia and in the presence of endothelial IR/IGF-1R (c), VEGF, eNOS, and ET-1 are increased, leading to extra-retinal neovascularization. Under conditions of relative hypoxia and in the absence of endothelial IR/IGF-1R (d), VEGF, eNOS, and ET-1 are reduced, possibly due to impaired HIF-1 activation or reduced $\mathrm{PI} 3 \mathrm{~K}$ activity related to IG/IGF-1R. Reduced neovascularization results from less IR/IGF-IR input.

\section{A role for insulin and IGF-1 in retinal neovascularization}

In this issue of the JCI, Kondo and colleagues (6) have utilized a clever genetic system in mice to probe the role of insulin and IGF signaling in this experimental model of relative hypoxia. They use two different genetically altered lines of mice, one of which contains an insulin receptor (IR) gene that can be deleted in the presence of Cre recombinase (7), resulting in an absence of the IR in all cells that have expressed the recombinase, and a similar line that contains an IGF-1 receptor (IGF-1R) gene that can be deleted in the presence of the recombinase (8). Experimental animals are bred to be homozygous for either of the modified receptor genes and to carry a transgene that expresses Cre recombinase under the control of the Tie-2 promoter/enhancer (9). The endothelium in these animals is systemically devoid of the IR or IGF-IR, and referred to as vascular endothelial insulin receptor knockout (VENIRKO) and vascular endothelial insulin-like growth factor receptor knockout (VENIFARKO), respectively. While neither Kondo et al. (6) nor Vicent et al. (10) confirm the loss of IR or IGF-1R proteins, it is presumed that the loss is nearly complete given the severe reduction in message detected by quantitative PCR amplification of endothelial cell RNA from these mice and the careful description of the Tie2-Cre transgenic line (9). The results are quite interesting.

First, Kondo (6) and Vicent (10) found no developmental or physiological consequences of IR or IGF-1R loss in endothelium; i.e., no gross or histological changes in the vasculature, and no metabolic changes such as those seen in subsets of the tissue-specific knockouts for each of these receptors (reviewed in ref. 11). However, when young mice are put through the relative hypoxia protocol there are distinct difwith an absence of the IR or IGF-1R in the endothelium. They find reduced neovascularization, and concomitantly ferences between control mice and those less immunoreactivity for markers of neovascularization including, VEGF, eNOS, and ET- 1 in VENIRKO and to a lesser extent in VENIFARKO mice, compared to wild-type mice. One cannot exclude the possibility that the reduction of VEGF, eNOS, and ET-1 is simply because there are fewer endothelial cells to express these proteins. Nonetheless, this result is interesting because deficiencies in the IR and the IGF-1R signaling pathways are critically linked to metabolic defects in Type I and Type II diabetes and insulin resistance - disease states where neovascularization is a frequent complication.

\section{New findings beget new questions}

The question is, what are the normal functions of the IR and IGF- $1 R$ in the retina? They probably do not regulate acute glucose utilization as they do in skeletal muscle, liver, and fat because their activity is constant under physiological conditions (12). In the CNS, the insulin and IGF signaling pathways stimulate embryonic growth, and loss of function mutations cause developmental eye defects in Drosophila (13). In retinal endothelial cells, insulin stimulates mitogenesis and insulin transport $(14,15)$. Hence, it is possible that the IR and IGF-1R in the retina function largely to provide trophic stimuli for maintenance of cell numbers. Therefore, signaling via factors upregulated by HIFs may intersect or augment the IR and IGF-1R pathways to promote neovascularization. The articles by Kondo et al. (6) and Vicent et al. (10) demonstrate that, from a developmental standpoint, endothelial cell insulin and IGF signaling are unnecessary, and consequences due to loss of signaling through these receptors may only be observed in response to some perturbation or injury. The neovascularization described by Kondo et al. presumably requires increased proliferation and/or survival of endothelial cell progenitors, so it is not surprising that disruption of the insulin and IGF signaling pathways results in less neovascularization. It would be helpful to know if the reduced vascularization in the knockout mice resulted from less proliferation or 
more apoptosis. The deciphering of downstream signals such as the activity of PI3K in the endothelium in response to relative hypoxia might also shed some light on this provocative result.

\section{Looking forward therapeutically}

The observations that loss of the IR and IGF-1R signaling pathways appear to counter the response to relative hypoxia and do not alter vasculogenesis in the absence of relative hypoxia suggest that it is not insulin and IGF-1 signaling in endothelial cells alone that promotes neovascularization in diabetes. It also prompts us to look to other cell types in the retina for a response to diminished insulin action. The experiments of Kondo et al. (6) do not address the role of retinal glia or neurons in vascular regulation. Answers to these questions will directly impact therapeutic choices for diabetic patients. Kondo et al. suggest that, in diabetes, inhibition of retinal insulin or IGF-1 signaling in the eye might be beneficial, however, in the context of what is understood to be systemically reduced insulin signaling in these disease states, therapeutic blocking of insulin signaling appears counterintuitive. Identification of specific molecules at the intersection of the HIF-1 and insulin and IGF-1 signals, as well as a thorough understanding of how the varied cell types in the retina respond to the diabetic state, will necessarily precede therapeutic trials to prevent loss of vision.

1. Provis, J.M., et al. 1997. Development of the human retinal vasculature: cellular relations and VEGF expression. Exp. Eye. Res. 65:555-568.

2. Neely, K.A., and Gardner, T.W. 1998. Ocular neovascularization: clarifying complex interactions. Am. J. Pathol. 153:665-670.

3. Grant, M.B., et al. 2002. Adult hematopoietic stem cells provide functional hemangioblast activity during retinal neovascularization. Nat. Med. 8:607-612.

4. Smith, L.E., et al. 1994. Oxygen-induced retinopathy in the mouse. Invest. Ophthalmol. Vis. Sci. 35:101-111.

5. Smith, L.E., et al. 1999. Regulation of vascular endothelial growth factor-dependent retinal neovascularization by insulin-like growth factor-1 receptor. Nat. Med. 5:1390-1395.

6. Kondo, T., et al. 2003. Knockout of insulin and IGF-1 receptors on vascular endothelial cells protects against retinal neovascularization. J. Clin. Invest. 111:1835-1842. doi:10.1172/JCI200317455.
7. Kulkarni, R.N., et al. 1999. Tissue-specific knockout of the insulin receptor in pancreatic beta cells creates an insulin secretory defect similar to that in type 2 diabetes. Cell. 96:329-339

8. Holzenberger, M., et al. 2003. IGF-1 receptor regulates lifespan and resistance to oxidative stress in mice. Nature. 421:182-187.

9. Kisanuki, Y.Y., et al. 2001. Tie2-Cre transgenic mice: a new model for endothelial cell-lineage analysis in vivo. Dev. Biol. 230:230-242.

10. Vicent, D., et al. 2003. The role of endothelial insulin signaling in the regulation of vascular tone and insulin resistance. J. Clin. Invest. 111:1373-1380. doi:10.1172/JCI200315211.

11. Kahn, C.R., Bruning, J.C., Michael, M.D., and Kulkarni, R.N. 2000. Knockout mice challenge our concepts of glucose homeostasis and the pathogenesis of diabetes mellitus. J. Pediatr. Endocrinol. Metab. 13:1377-1384.

12. Reiter, C.E.N., and Gardner, T.W. 2003. Retinal insulin and insulin signaling: implications for diabetic retinopathy. Prog. Ret. Eye Res. 22:545-562.

13. Broglio, W., et al. 2001. An evolutionarily conserved function of the Drosophila insulin receptor and insulin-like peptides in growth control. Curr. Biol. 11:213-221.

14. King, G.L., Goodman, A.D., Buzney, S., Moses, A., and Kahn, C.R. 1985. Receptors and growth-promoting effects of insulin and insulinlike growth factors on cells from bovine retinal capillaries and aorta. J. Clin. Invest. 75:1028-1036.

15. Stitt, A.W., Anderson, H.R., Gardiner, T.A., Bailie, J.R., and Archer, D.B. 1994. Receptor-mediated endocytosis and intracellular trafficking of insulin and low-density lipoprotein by retinal vascular endothelial cells. Invest. Ophthalmol. Vis. Sci. 35:3384-3392.

\section{Tolerance: Of mice and men}

\section{David H. Sachs}

Transplantation Biology Research Center, Massachusetts General Hospital, Boston, Massachusetts, USA

\begin{abstract}
Little is known about the effect of an individual's immune history on his or her response to an allogeneic tissue transplant. An important study (see the related article beginning on page 1887) now reveals that individuals harboring virally-induced memory $T$ cells that are cross reactive with donor alloantigen are resistant to conventional strategies designed to induce transplant tolerance.
\end{abstract}

\section{J. Clin. Invest. 111:1819-1821 (2003). doi:10.1172/JCI200318926.}

Enormous progress has been made in the field of transplantation during the past three decades, due in large part to the availability of effective

\footnotetext{
Address correspondence to: David H. Sachs, Transplantation Biology Research Center, Massachusetts General Hospital, MGH East, Building 149-9019, 149 13th Street, Boston, Massachusetts 02129, USA. Phone: (617) 726-4065; Fax: (617) 726-4067; E-mail: sachs@helix.mgh.harvard.edu. Conflict of interest: The author has declared that no conflict of interest exists.
}

immunosuppressive drugs. Although all of these agents suppress the immune response nonspecifically with respect to antigen, the most effective ones exhibit sufficient selectivity so that rejection can be avoided without undue compromise of the host's ability to respond to microbial pathogens. Nevertheless, patients on immunosuppressive medications are constantly walking a tightrope between the consequences of too little suppression (i.e., rejection) and of too much suppression (infections or cancer) of their immune system. In addition, even in patients without complications due to their immunosuppression, there is an inexorable loss of transplanted organs due to chronic rejection at a rate of approximately $5 \%$ per year (1).

For these reasons, ever since the description of acquired tolerance to allografts in mice by Medawar and colleagues appeared in 1953 (2), a major goal of both clinicians and immunologists in the field of transplantation has been the induction of tolerance in transplant recipients. What has been most frustrating about this quest has been the fact that a very large number of successful approaches to the induction of tolerance have been reported in rodent models, but have failed when attempted in large animals, especially in nonhuman primates and in humans (Table 1). Indeed, as clinical results of organ transplants using standard immunosuppression are so good, at least in the short term, many clinicians are no longer interested in 